\title{
Review on Multi-Field Application of Dielectric Resonator Antenna
}

\author{
Abinaya Nallathambi ${ }^{1,2}$ and Rajesh Narayana Perumal ${ }^{2 *}$ \\ ${ }^{1}$ Department of Biomedical Engineering, SSN College of Engineering, India \\ ${ }^{2}$ Centre for Radiation Environment Science and Technology, SSN College of Engineering, India
}

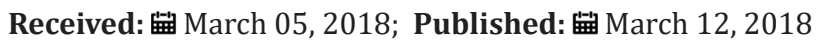

*Corresponding author: Rajesh Narayana Perumal, Centre for Radiation Environment Science and Technology, SSN College of Engineering, Kalavakkam 603110, India, Email: rajeshnp@ssn.edu.in

\begin{abstract}
The major aim of this paper is to collate the information on application oriented performance of DRA. Here we dominantly discussed on multi functional performance of antenna as a package material along as oscillator and filter and multi aspects of transparent DRA. A glimpse on On-Chip-DRA considering difficulty of the process to encourage the knowledge to overcome discrepancy. A note on Material and geometry influence in different application including RADAR, RFID is discussed. The advantage of small ground plane is discussed and markedly we produced details on Biomedical application of DRA that contrived a significant distinct compared with other Review articles of DRA.
\end{abstract}

Keywords: DRA- Dielectric Resonator Antenna

\section{Introduction}

The dielectric resonator of shielded nature acted as filter and oscillator with requirement of high $Q$ value later the resonator where found to radiate in incomplete conducting environment which was noticed initially by Richtmyer in early 1939 [1] later Long et al. [2] studied the DRA both theoretically and experimentally since then piecemeal analysis on identifying Q-factor by Gastine et al. [3]. Constructing DRA as a small antenna by Sager, Tisi [4], evaluation of different modes and supporting them with numerical results by Van Bladel [5,6] were reported. The First review of DRA by Rajesh K Mongia et al. [7], contributed pristine simplified collated information on modes, frequency based designing for various shapes and conditions like isolated and metallic plane supported DRA. Dielectric resonator antenna appealing engineers due to their mechanical simplicity, nil inherent conductor loss, easy optimization and different characteristics of radiation was obtained for excitation of different modes due to unique field distribution.

DRA is under research for 3 decades, Researchers contributed enormous knowledge including more than 900 patents and multiple review paper [8-14] and 2 excellent books [15,16]. In India Dr. Debatosh guha and Cohort done lot of work in different feed mechanism and new mode excitation and dealt with fundamentals of DRA In this review we are not including this because most topics are already covered in previous reviews. Hence major focus is on biomedical application of DRA along with glimpse of few new structures and other applications of DRA.

Multi-functionality of Dielectric Resonator antenna: DRA inherent nature made it to be used for multiple activities in individual and also combined, such systems are discussed below:

Packaging and Dual Circuitry Function: KW Leung originally proposed the packaging application of Hollow RDRA following PoPov patented the idea of circular and linear polarization of packaged integrated DRA, Eng Kock Lim and Kwok Wa Leung [17] implemented this concept in integrating the active and passive elements into the hollow structure, He concentrated on rectangular DRA (RDRA) due to its mechanical simplicity and three degree design freedom to obtain varied parametric selection, Keller's limitation to integrate the active element to the DRA overcame by them and it act as a antenna as well as a cover, this offer wide advantage like improving the noise reduction. Following in $[18,19]$ demonstrated the relevance to both antenna well as filter and also as Oscillator and proved the dual functional capability of DR along with hollow structure they are exhibiting the tri functionality with effect of packaging. We knew the contrast requirement of antenna, filter and oscillator this was overcame by exciting DR in two 
different modes namely TE0 $1 \delta$ and HEM $11 \delta$ that are orthogonal to each other thus two integrated circuits can be can separately tuned, and the construction in [19] is design to tune the filter and such multifunctional DRAF is suited for GPS Device.

Aesthetic Antenna: KW Leung et al. [11] given enormous contribution to DRA, in review on the fundamentals like resonant frequency calculation, and major details on transparent antennas that are commercially purchased apple and swan shaped glass ware that contains lead oxide. This investigation serves as a proof that the decorative items can act as an efficient antenna; this idea is more psychologically inspiring. These are also applied in vehicles, building windows, computer monitors, solar photovoltaic panels and also as aesthetics, but on the defect note these transparent antenna made up of material like glass and acrylic act as substrate over which conductive film is printed previously using good conductors like copper but in order to retain the optical transparency transparent materials like indium tin oxide (ITO), fluorine-doped tin oxide (FTO) and AgHT-8,coated on the DRA like a patch this allows current to flow and thus the material act as an antenna but they are not efficient conductors comparatively [20].

After the proposal EH Lim [21] and KW Leung [22] transparent glass (Pyrex) DRA devoid conducting parts to resonate, and a hollow rectangular glass DRA that additionally serves as a light cover was investigated, Ceaselessly they exhibited its capacity on [23] focusing lens and antenna incorporated with solar panel of hemispherical shape. Recently [24] transparent low-permittivity polymer (acrylic) is placed on top of a silicon solar cell adhered with RTV silicone, glass protection with metals strips to tune to dual, narrow, wide and multi bands provided suits X-band satellite communications application.

\section{Material and Structure Value in Application}

Material and geometry play the major role in determining the performance of antenna, apart from regular Cylindrical, Rectangle, Hemisphere new shapes like pentagon, tree, tilted, semi hollow cylinder, hollow cylinder and rectangle, pentagon are also investigated. Materials like Pyrex, alumina ceramics- (ZST, BST, and PZT etc.), polymers like acrylic sheet and many, fabrics like cotton and even liquid like ethyl acetate, water, and oil are investigated for different purpose like multiple band, wideband frequency, among them few applications are discussed below. A pentagon shaped DRA of both regular and irregular characteristics successfully excited by a single slot by Sathis K Sharma et al. [25] for wide band and multiband with resonant frequency of 2.42-2.61 GHz, 3.15-4.05 $\mathrm{GHz}$ and 5-5.65 GHz respectively. L. Huitema used a technique on masking the part of DR with metal coating for the desired ultrahigh frequency of bands IV, V and GSM this reduce the cost of machining to modify the shape and also relies on the image theory technique.

Further discussed on a design of DRA using magneto-dielectric material which posses both magnetic and dielectric characteristics
( $\varepsilon r=6.5, \mu r=3.7$ ) on improving the impedance bandwidth, and covers the required UHF antenna [26]. Xiao-Chuan Wang et al. design of low profile RDRA in CNSS Bands $1 \& 3$ with right hand circular polarization of central frequency $\mathrm{f} 1=1.268 \mathrm{GHz}, \mathrm{f} 2=1.561$ $\mathrm{GHz}$, using ceramic material by the cross arm etched in ground plane [27]. Y. Coulibaly et al. [28] designed antenna of $60 \mathrm{GHZ}$ frequency which is apt for underground communication, but high gain is required to suit so CDRA was added with a superstrate which proved to enhance the gain that makes the antenna much suitable for the underground communication. In H Fayad [29] and P Record reported a semi hollow cylindrical shape of DRA. Difficult to manufacturing due to mechanical constrain of ceramics, the removal of the centre part will increase the bandwidth but the air gap create different resonant frequency thus the shape here contribute the dual frequency, Generally such gaps are filled with liquid or water depend on application requirement, there are also some investigation where the entire DR Material itself Liquid based, this is detailed in following section.

Liquid DRA: Liquid DRA is trending now due its less cost and high radiation efficiency and high permittivity in turn related to the miniature of device, Zhe Chen et al. [30] designed a combination of two different state of material (hybrid) which is of K9 glass and Liquid ethyl acetate, that is fill in a container fabricated using VEROBLUE RGD 840 by 3D printing and bottom is sealed with glue, to reconfigure the pattern of radiation of DRA. This is well suited to the system requiring static changes but there is lot of issues faced due to the hybridism so the authors in [31] reported the design with elimination of glass and clubbed two container. Recently in [32] investigated the hybrid effect of oil and water, interestingly to make the antenna tunable they used the principle of communicating vessels, however more investigation is required on this topic to improve their utilization in real world application.

Radar: Here authors proposed a design that suitable radiate in frequency of radar thus considered for radar application. Idris Messaoudene et al. [33] used stacked DRA technique with different material like TMM10i ( $\varepsilon=9.8$ ) and BST (Ba0.8Sr0.2Ti03) of $\varepsilon=250$ for bottom and top respectively, similarly Massinissa Belazzoug et al. [34] is also designed two half cylindrical DRA that is of compact size excited by probe covers a large band including WLAN, X-band, and C-band with high radiation efficiency and becomes suitable for portable devices, body-centric Wireless Communications, and RADAR applications.

RFID: Hend A. Malhat et al. [35] investigated an elliptical dielectric resonator antenna (EDRA) for handheld RFID reader a trending application that used in production, logistics, supply chain management and asset tracking. The Far field coupling of RFID relies on the principle of Electromagnetic waves coupling that require antenna covering any one of the frequency bands 100$500 \mathrm{kHz}, 13.56 \mathrm{MHz}, 860-960 \mathrm{MHz}, 2.45 \mathrm{GHz}$ and $5.8 \mathrm{GHz}$, their proposed design that has achieved $66.7 \mathrm{MHz}$ impedance bandwidth 
(for $\mathrm{S} 11<-10 \mathrm{~dB}$ ) at resonant frequency of $5.8 \mathrm{GHz}$ by using material ( $\varepsilon r=12$ ) with coaxial probe feed in free space and using, the finite element method (FEM) and the finite integration technique (FIT), CAD model of device and human hand to simulate the investigation the performance of antenna in real environment.

RF Energy Harvesting: Advantageous DRA cannot be exempted from RF energy harvesting, few investigated on gain, power absorbed and incident angle and optimizing the parametric to identify best configuration that suits the purpose [36,37].

On Chip Antenna: On chip is the futuristic approach since a single chip itself act as device many trialed on this few details are as follows, Mai O. Sallam et al. [38] illustrated a method of fabricating the DRA in micro range using micromachining technique, JP Guzman et al. [39] offered a complete solution on packaging CDRA of $60 \mathrm{GHZ}$ frequency using laser cutting tool over the Alumina material, flipping it over substrate another technique is integration of silicon chip and silicon active element. This idea offers efficient packaging say the entire device size itself in $5 \mathrm{~mm} \times 5 \mathrm{~mm}$. the review on these on-chip DRA is also given in [40]. However, first the integration of DRA on silicon substrate chip was performed by PV Bijumon [41,42] for 27GHZ.Here author along with general consideration suggested careful selection of size and shape and manufacturing technique also concerned with the choice of material, and even the glue that was used to integrate the DRA with the chip which have effect in permittivity of DR. one could evidence that how precise information has to be considered with developing a process. Glue less integration is also possible, LTCC and CMOS technique also available for on-chip manufacturing.

Nano Antenna: Nano Antennas obviously operate at optical frequency, Chengjun Zou et al. [43] presented a review on the Nano DRA with sufficient basic concept discussed with the start of Nano DRA efficiency over the plasmonic antenna to the end of reasons of resonance break down and little description on future scope of field and related research work that has to be forwarded to implement the approach in real world application. A different structured Nano antenna is proposed in [44] that operate at $189 \mathrm{THz}-194 \mathrm{THz}$ with bandwidth of $2.58 \%$ and stand as a suitable candidate for optical applications in the C-band window of optical communications.

Small Ground: YM Pan et al. $[45,46]$ investigated on the small ground plane and successfully registered the outcome without portentous effect on impedance matching, here the patch which is used for excitation is acting as ground this is much useful along if packaging application is considered, which is quite complex but not impossible. If such case is investigated the future of DRA apart from being a part of device will become entire device. In future the research might be in the name of Dielectric Resonator Device application.

Biomedical Application: A candidate like DRA with multiple inherent advantage is much suited for biomedical application. The radiation efficiency fit for telemetry application, This antenna is capable in producing millimeter waves involve in cell regeneration where so far this topic is not addressed using DRA and THz waves are hot topic in imaging and tumor detection, detail discussion is as follows.

Wireless BAN: Concepts of Body area network, implantable antenna, wearable antenna equally interest scientist as in MIMO and LTE application, DRA also not exceptional to it. L. Nageswara Rao [47] designed a serpentine slot that is stacked with DRA and using single micro strip generate the frequency in MICS \&ISM bands for medical application though there is lots of work has to be proceeded, this is a good start.

Textile based DRA for wearable Antenna: Mian Shahzad Iqbal, Karu P Esselle [48] also worked on wearable DRA which is simulated with muscle phantom with consideration of worn clothes using 3 different dielectrics mounted and excited using probe feed, antenna parameters are discussed but devoid SAR characterization which an important part when comes to interaction with human is lacking here. However Textile based DRA has been already patented. In SHH Mashhadi et al. [49,50] investigated on the textile based DRA, it is said so because the DRA of Bow tie Shape (TMM10i, $\varepsilon=9.8$ ), is placed above a fabric substrate here it is knitted cotton with buckram and metallic ground plane with a micro strip feed mechanism both of Aluminum fiber conductive yarn, the same configuration treated on human body and found acceptance. From his trial he contributed some factors that has to be consider for future application to develop that into device, those are as follows:

a) Choice of textile geometry such as it improves stability and also withstands any tough condition.

b) Matching techniques.

c) Studies on different fabric that offer better performance.

d) Suggested the kind of phantom for investigation.

e) Apart from all the above the power source which is a separate topic of research also should be considered to make compact device.

DRA in Imaging Application: Terahertz (THz) technologies apart from security screening and high-speed wireless data communications applies to the biomedical imaging [51,52]. When concerned to the biomedical application part THz wave is nonionizing, very safe technology for human tissues as compared with the X-ray and can image with high precision to the level of DNA, amino acids, proteins. Hence engineers are highly interested in device fabrication for $\mathrm{THz}$ frequency, with optimal gain so that there is pronounced reduction of noise is possible, since THZ radiated in small size antenna along with circuitry, there is requirement of integrating techniques like CMOS, however CMOS on-chip antennas exhibit low antenna gain and limited radiation 
efficiency due to lossy silicon substrates and unfriendly metal back end of lines (BEOL). Though many techniques have been proposed to improve the on-chip antenna performance. The fabrication process involves higher complexity.

So there was a trial proposed with believe that the DRA due to the appealing inherent quality will solve the issue. In [53] using 0.18- $\mu \mathrm{m}$ CMOS technology on low dielectric material with a high-Z silicon DR of rectangular shape is investigated, and it gave a considerable gain compared to the other antenna this is due to the directivity of DRA, similarly in [54], the flipped DR thermocompressively bonded to the CMOS chip, both technique realized to be worth for practical application in improving gain, furthermore the antenna with reduced size and permittivity is most effective in performance and also have the advantage of low cost and easy fabrication. So it is believed that $\mathrm{THz}$ imaging using DRA will replace the imaging technique.

Replacing component of MRI with DRA: The principle of MRI is based on the alignment of hydrogen nuclei due to strong magnetic field, and thus the tissue emit RF waves which is captured by the $\mathrm{RF}$ coil place near the object to be imagined, This part is replaced with DRA which take over the transceiver function in Transverse electric (TE) $01 \delta$ mode and hybrid electromagnetic (HEM) $11 \delta$ in ultrahigh field MRI. and two methods of double-tuning the ceramic resonator to proton $(298 \mathrm{MHz})$ and fluorine $(280 \mathrm{MHz})$ resonant frequencies at 7T this is reported in [55] and the major issue of MRI like B1 in homogeneities which is the defect of varied dielectric and conductive property of tissues of human body, previously it was compensated by placing dielectric pads at interface of patient and transmit coil this shows good improvement in homogeneity in body and neuroimaging at high field and Neuro-imaging in ultrahigh field MRI [56,57], and to improve signal intensity in the cervical spine at $3 \mathrm{~T}$ and in the inner ear at $7 \mathrm{~T}$. The solution to this issue is addressed in [58].

The inherent isolation capacity of DRA avoids the decoupling circuit, In addition to it the light weight and size which inversely related to the permittivity made it possible for miniature MRI equipment with little more consideration in advancement with respect to the packaging and the safety precaution that should be taken on the effectstrong magnetic condition this can very well act as a portable MRI, with much reduced in homogeneities. It even avoids impedance matching with skin. For more experimental details refer [59]. By compensating the major component, with DRA is available with varied material with variety of configuration which is flexible by the preparation methods. An antenna configuration proposed in [60] is 4-by10 DRA array. The antenna size, including the feed and the waveguide to SIG transition, is $34 \mathrm{~mm} \times 5 \mathrm{~mm}$, with high gain of $19 \mathrm{~dB}$ is suitable for imaging application.

DRA in Tumour Detection: Wei Huang and Ahmed A. Kishk in [61], designed a stacked DRA Array of in size of millimeter is by using the transmitting and receiving capacity of antenna in microwave range, this is designed so as the microwave emit the most towards the tissue and thus from varied nature of tissue the EM waves is reflected back to the antenna which is received by DRA thus it act as sensing device. One such investigation using H-shaped DR is presented in [62]. Some patents are also filed in the same category of application for e.g. refer [63]. With the knowledge of integrating DRA to sensors many application beyond biomedical can be performed like sensing the temperature, corrosion and pressure in environment some DR even act as humidity sensor and in DRA is investigated for concrete characterization. That sounds similar to the ultrasound device that is used to find cracks in building, but notice that both the principle of operation is entirely different; here this is mentioned as a comparative or even a compensative illustration.

\section{Conclusion}

Ginormous works have been done and happening in Dielectric resonator antenna to make it marketable product in various application due to its advanced nature, this article mostly covered the investigation based on application up-to-date. Intentionally general information is elaborated than the technical details due to already available bunch of technical in previous reviews. The major aim of this paper is to create a curiosity and multiple ways to the engineers to develop DRA based device for the future.

\section{References}

1. Richtmyer RD (1939) Dielectric Resonators. J Appl Phys 10: pp. 391-398.

2. Long SA, McAllister M, Shen LC (1983) The Resonant Cylindrical Dielectric Cavity Antenna. IEEE Tans Ant Propagat AP-31.

3. Gastine M, Courtois L, Dormann JJ (1967) Electromagnetic Resonances of Free Dielectric Spheres. IEEE Trans Microwave Theory Tech MTT15(12): 694-700.

4. Sager O, Tisi F (1968) On Eigenmodes and Forced Resonance-Modes of Dielectric Spheres. Proc IEEE 56(9): 1593-1594.

5. Van Bladel J (1975) On the Resonances of a Dielectric Resonator of Very High Permittivity. IEEE Trans. Microwave Theory Tech MTT-23(2): 199208.

6. Van Bladel J (1975) The Excitation of Dielectric Resonators of Very High Permittivity. IEEE Trans Microwave Theory Tech MTT-23(2): 208-217.

7. Rajesh KM, Prakash B (1994) Dielectric resonator antenna-a review and general design relation for resonant frequency and bandwidth. International Journal of Microwave and millimetre wave computer aided engineering 4(3): 230-247.

8. Petosa A, Ittipiboon A, Antar YMM, Roscoe D, Cuhaci M (1998) Recent advances in dielectric resonator antenna technology. IEEE Antennas Propag. Mag 40(3): 35-48.

9. Antar YMM (2008) Antennas for wireless communication: Recent advances using dielectric resonators. IET Circuits Devices Syst 2(1): 133-138.

10. Petosa A, Ittipiboon A (2010) Dielectric resonator antennas: A historical review and the current state of the art. IEEE Antennas Propag Mag 52(5): 91-116.

11. Leung KW, Lim EH, Fang XS (2012) Dielectric resonator antennas: From the basic to the aesthetic. Proc IEEE 100(7): 2181-2193. 
12. Sounik KKD, Taimoor Khan, Asok De (2016) Dielectric resonator antennas: An application oriented survey. Int J RF Microw Comput Aided Eng p.23.

13. Chengjun Zou, Withawat W, Madhu B, Sharath S, Christophe F (2017) Dielectric Resonator Nano-antennas A review of the theoretical background, design examples, prospects, and challenges. IEEE Antennas \& Propagation Magazine 59(6): 30-42.

14. Sounik KKD, Taimoor Khan, Yahia MMA (2018) A state-of-art review on performance improvement of dielectric resonator antennas. International Journal of RF Microwave Computed Aided Eng e21270.

15. (2003) Dielectric Resonator Antennas. (In Luk KM, Leung KW, Eds: Baldock) Research Studies Press, UK.

16. (2007) Dielectric Resonator Antenna Handbook, A Petosa, Norwood, MA: Artech House,UK.

17. (2016) Design of Hollow Cylindrical Dielectric Resonator Antenna Excited By Micro-strip Lines, International Journal of Emerging Technology and Advanced Engineering.

18. Eng Hock Lim, Kwok Wa Leung (2006) Novel Application of the Hollow Dielectric Resonator Antenna as a Packaging Cover. IEEE Transactions on Antennas and Propagation. 54(2): 484-487.

19. Eng Hock Lim, Kwok Wa Leung (2008) Use of the Dielectric Resonator Antenna as a Filter Element. IEEE Transactions on Antennas and Propagation 56(1): 5-10

20. Eng Hock Lim, Kwok Wa Leung (2007) Novel Utilization of the Dielectric Resonator Antennas an Oscillator Load. IEEE Transactions on Antennas and Propagation 55(10): 2686-2691.

21. Kwok W, Leung, Yong M Pan, Xiao S Fang, Eng H Lim, et al. (2013) Dual-Function Radiating Glass for Antennas and Light Covers-Part I: Omni directional Glass Dielectric Resonator Antennas, Member, IEEE, Transactions on Antennas And Propagation 61(2): 578 - 586

22. EH Lim, KW Leung (2010) Transparent dielectric resonator antennas for optical applications, IEEE Trans. Antennas Propag 58(4): 1054-1059.

23. Eng Hock Lim, Kwok Wa Leung (2010) Transparent Dielectric Resonator Antennas for Optical Applications. IEEE transactions on antennas and propagation 58(4): 1054 - 1059

24. Atabak Rashidian, Lotfollah Shafai, Cyrus Shafai (2017) Miniaturized Transparent Metallo-Dielectric Resonator Antennas Integrated with Amorphous Silicon Solar Cells. IEEE Transactions on Antennas and Propagation 65(5): 2265 - 2275

25. SatishK, Sharma, Manveer K Brar (2013) Aperture-Coupled Pentagon Shape Dielectric Resonator Antennas Providing Wideband And Multiband Performance. Microwave and Optical Technology Letters 55(2): 395-400

26. L Huitema, M Koubeissi, C.Decroze, T. Monediere (2010) Handheld Dielectric Resonator Antenna for Ultra Wideband Applications. IEEE.

27. Xiao Chuan Wang, Lin Sun, Xian Long Lu, Sen Liang, Wen Zhong Lu (2017) Single-Feed Dual-Band Circularly Polarized Dielectric Resonator Antenna for CNSS Applications. IEEE 65(8): 4283-4287.

28. Y Coulibaly, M Nedil, Larbi Talbi, T A Denidni (2010) High Gain Cylindrical Dielectric Resonator with Superstrate for Broadband Millimeter-Wave Underground Mining Communications. $14^{\text {th }}$ International Symposium on Antenna Technology and Applied Electromagnetics [ANTEM] and the American Electromagnetics Conference [AMEREM]

29. Fayad, Ezzeldin A, Soliman P Record (2015) Multi-Feed Dielectric Resonator Antenna With Reconfigurable Radiation Pattern. H, Progress In Electromagnetics Research, PIER 76, 2007and, IEEE TRANSACTIONS ON ANTENNAS AND PROPAGATION 63(8): 2157-2164.

30. Zhe Chen, Hang Wong, Jun Xiang (2017) Polarization-Reconfigurable Liquid Dielectric Resonator Antenna with 3D Printing Fabrication. IEEE.
31. Gaosheng Li, Gui Gao , Li-an Bian , Zhonghao Lu, and Chaoyun Song A Tunable Antenna with the Combination of Two Kinds of Liquid Materials. 32(11): 966-973.

32. Idris Messaoudene,Farouk Chetouah, Massinissa Belazzoug (2017) Compact Rectangular DRA with High Permittivity Stacked Resonator for RADAR Applications. IEEE.

33. Massinissa Belazzoug, Idris Messaoudene, Salih Aidel, Youcef Braham Chaouche, et al. (2017) Farouk Chetouah A Compact MIMO Antenna for WLAN and RADAR Applications. IEEE.

34. Hend A, Malhat, Saber H Zainud Deen, Kamal H Awadalla (2015) Circular Polarized Dielectric Resonator Antenna for Portable RFID Reader Using a Single Feed. IEEE.

35. Ashoor AZ, Ramahi OM (2015) Prog Electromagn Dielectric Resonator Antenna Arrays for Microwave Energy Harvesting and Far-Field Wireless Power Transfer. Res C 59: 89-99.

36. AA Masius, YC Wong, KT Lau (2017) Miniature High Gain Slot-fed Rectangular Dielectric Resonator Antenna for IoT RF Energy Harvesting. International Journal of Electronics and Communications.

37. Mai O Sallam, Mohamed Serry, Sherif Sedky, Atif Shamim, Walter De Raedt (2015) Micromachined On-Chip Dielectric Resonator Antenna Operating at $60 \mathrm{GHz}$. IEEE TRANSACTIONS ON ANTENNAS AND PROPAGATION 63(8): 3410-3416.

38. JP Guzman, SA Muhammad, M Ney, C Person (2012) Cylindrical Dielectric Resonator Antenna excited by on-chip integrated slot for a completely packaged $60 \mathrm{GHz}$ front-end solution. Amsterdam, The Netherlands.

39.S Keyrouz, D.Caratelli, (2016) Dielectric Resonator Antennas: Basic Concepts, Design Guidelines, and Recent Developments at MillimeterWave Frequencies, International Journal of Antennas and Propagation p. 20.

40. PV Bijumon, AP Freunddorfer, M Sayer, Y M M Antar (2007) High Gain On-Chip Dielectric Resonator Antennas Using Silicon Technology for Millimeter Wave Wireless Links. Canadian Conference on Electrical and computer Engineering pp. 804-807

41. P V Bijumon, YMM Antar, AP Freundorfer, M Sayer (2008) Dielectric Resonator Antenna on Silicon Substrate for System On-Chip, IEEE Transactions On Antennas And Propagation 56(11): 3404-3410.

42. Chengjun Zou, Withawat Withayachumnankul, Madhu Bhaskaran, Sharath Sriram, Christophe Fumeaux (2017) Dielectric Resonator Nanoantennas A review of the theoretical background, design examples prospects, and challenges. IEEE Antennas \& Propagation Magazine 59(6): 30-42.

43. Waleed Tariq Sethi, Hamsakutty Vettikalladi, Habib Fathallah,Mohamed himdi (2016) lx2 Equilateral Triangular Dielectric Resonator Nantenna Array for Optical.

44.Y M Pan, K W Leung, K Lu (2013) Compact quasi-isotropic dielectric resonator antenna with small ground plane. IEEE Trans. Antennas Propag 62(2): 577-585.

45. Y M Pan, K W Leung, L Guo (2017) Compact Laterally Radiating Dielectric Resonator Antenna With Small Ground Plane.IEEE Transactions on Antennas and Propagation 65(8): 4305-4310.

46. L. Nageswara Rao (2014) A Cylindrical Dielectric Resonator Antenna for Wireless Body Area Networks, International Journal of Microwaves Applications 3(1):7-11.

47. Mian Shahzad Iqbal, Karu P Esselle (2014) A Compact Wideband Dielectric Resonator Antenna for On-Body Applications. IEEE.

48. S H H Mashhadi, Z Wu, LZ Thamae (2010) Investigation Of A Wearable Broadband Textile Dielectric Resonator Antenna. IEEE.

49. S H H Mashhadi, Z Wu, LZ Thamae, S M Y Abbas (2012) Transmission Characteristics of Wearable Broadband Textile Dielectric Resonator Antennas In Body Area Network. IEEE. 
50. P H Siegel (2002) Terahertz technology IEEE Trans. Microw. Theory Techn 50(3): 910-928.

51. P H Siegel Terahertz technology in biology and medicine. IEEE Trans. Microw. Theory Techn, 52(10): 2438-2447.

52. Chun- Hsing Li, Te-Yen Chiu (2017) 340GHz Low-Cost and High-Gain On-Chip Higher Order Mode Dielectric Resonator Antenna for $\mathrm{THz}$ Applications. IEEE 7(3): 284-294.

53. Te-Yen Chiu, Chun Wang, Wan-Ting Hsieh, Hsien-Jia Lin, Ta-Yeh Lin, et al. (2017) A 340-GHz High-Gain Flip-Chip Packaged Dielectric Resonator Antenna for THz Imaging Applications. IEEE 7(3): 284-294.

54. Aussenhofer SA, Webb AG (2013) High-permittivity solid ceramic resonators for high-field human MRI. NMR Biomed 26(11): 1555-1561.

55. Snaar JE, Teeuwisse WM, Versluis MJ, van Buchem MA, Kan HE (2011) Improvements in high-field localized MRS of the medial temporal lobe in humans using new deformable highdielectric materials. NMR Biomed 24(7): 873-879.

56. Teeuwisse WM, Brink WM, Webb AG (2012) Quantitative assessment of the effects of high-permittivity pads in 7 Tesla MRI of the brain. Magn Reson Med 67(5): 1285-1293.
57. Lu JY, Zhang X, Rutt BK (2013) A novel 7T transmit array using TE01 $\delta$ mode dielectric resonators. In Proceedings of the 21st Annual Meeting of ISMRM, Salt Lake City, Utah, USA 21:4376.

58. Aussenhofer SA, Webb AG (2014) An eight-channel transmit/receive array of TE01 mode high permittivity ceramic resonators for human imaging at 7T. J Magn Reson 243: 122-129.

59. Alireza Zandieh, Ahmed Shehata Abdellatif, Aidin Taeb, and Safieddin Safavi-Naeini (2017) High Resistivity Silicon DRA Array for MillimeterWave High Gain Applications IEEE.

60. Wei Huang, Ahmed A Kishk (2006) Design of Dielectric Resonator Sensor with Minimized Ground Plane Size for Microwave Breast Cancer Detection. IEEE.

61. Kenny Seungwoo Ryu, and Ahmed A. Kishk (2011) Evaluation of Dielectric Resonator Sensor for Near-Field Breast Tumor Detection. IEEE,transactions on antennas and propagation 59(10): 3738-3745.

62. Suhami (2017), United States, USPTO.

63. Sumyea Sabrin, Sergey Kharkovsky , Robert Salama (2017) Dielectric resonator antenna integrated sensors for characterization of concrete. International Conference on Sensing Technology (ICST).

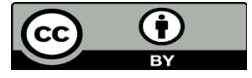

This work is licensed under Creative Commons Attribution 4.0 License

To Submit Your Article Click Here:

Submit Article

DOI: $10.32474 /$ OAJBEB.2018.01.000119

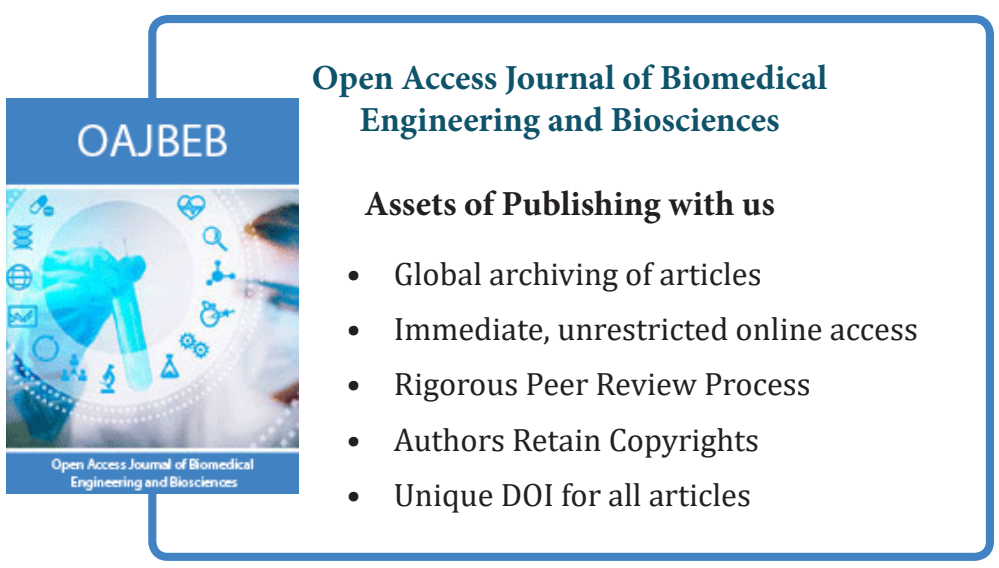

